\title{
Autosomal suppressors of sex-ratio in Drosophila mediopunctata
}

\author{
ANTONIO BERNARDO DE CARVALHO* \& LOUIS BERNARD KLACZKO† \\ Departamento de Genética, Instituto de Biologia, Universidade Federal do Rio de Janeiro, Caixa Postal 68011 , \\ CEP 21944, Rio de Janeiro, Brasil and TDepartamento de Genética e Evolução, Instituto de Biologia, Universidade \\ Estadual de Campinas, Caixa Postal 6109, CEP 13081/970, Campinas, SP, Brasil
}

\begin{abstract}
The sex-ratio trait has been described as the production of progenies with excess of females due to $\mathrm{X}$-linked meiotic drive in the parental males. This trait has a variable expression in Drosophila mediopunctata. We describe here the existence and chromosomal localization of autosomal suppressors of sex-ratio in this species. There are at least four such genes (one on each major autosome) and the strongest effect is localized on chromosome IV. These genes possibly result from the operation of 'Fisher's Principle'; a mechanism of Natural Selection leading to a 1:1 sex ratio.
\end{abstract}

Keywords: Drosophila mediopunctata, chromosomal analysis, evolution of sexual proportion, Fisher's Principle, sex-ratio, suppressors of meiotic drive.

\section{Introduction}

In several Drosophila species there is a trait known as 'sex-ratio' in which males carrying certain X chromosomes (generally called 'SR' and usually associated with chromosome inversions) produce progenies with a large excess of females (Gershenson, 1928; James \& Jaenike, 1990). For the two species investigated in this respect, the lack of males is caused by degeneration of Y-bearing spermatids during spermiogenesis (Policansky \& Ellison, 1970; Hauschteck-Jungen \& Maurer, 1976). Sex-ratio is thus a special case of meiotic drive which affects the sex chromosomes.

In theory, as a meiotically driven chromosome, SR tends to become fixed and this might cause population extinction due to the absence of males (Gershenson, 1928; Hamilton, 1967). However, stable SR polymorphisms are found in natural populations. Several factors appear to be involved in the stabilization of these polymorphisms, including natural selection against SR and modifier genes that suppress or attenuate the meiotic drive (Wallace, 1948; Beckenbach, 1991; Stalker, 1961).

Suppressor genes are expected on the Y chromosome because any Y-linked gene that increases its transmission rate will spread, unless associated with a large fertility loss (Thomsom \& Feldman, 1975). Powerful suppressors of this kind have been found in

*Correspondence.
D. paramelanica (Stalker, 1961), D. affinis (Voelker, 1972) and D. mediopunctata (Carvalho, 1989; Carvalho \& Klaczko, 1992, unpublished data).

Autosomal suppressors are expected to evolve in response to the spread of SR due to 'Fisher's Principle' (Fisher, 1930). In any sexually reproducing population, half of the genes come from each sex, irrespective of its rarity. If there is a rare sex such as males in SR bearing populations, it will be effectively more fertile as a result of a greater per capita contribution to the next generation. Consequently alleles directing the reproduction to this rare sex (as autosomal suppressors of SR do) will spread until the sex proportions are in equilibrium. Fisher's Principle is the most accepted explanation for the equivalence of sexual proportions but there is only one demonstration of its operation: a laboratory experiment with a fish (Conover \& Voorhees, 1990). Autosomal suppressors of sex-ratio may provide another system to study the evolution of sexual proportion, perhaps in nature. Their existence was suggested in $D$. paramelanica (Stalker, 1961) but a search for them in natural populations of $D$. pseudoobscura was unsuccessful (Policansky \& Dempsey, 1978; Beckenbach et al., 1982). More recently Cobbs $(1986,1987)$ and Cobbs et al. (1991) described modifiers of sexratio in one strain of $D$. pseudoobscura but, given the invariable sterility of the sons of SR/Y males in this species, these genes cannot be considered 'Fisherian suppressors'.

In D. mediopunctata the sex-ratio trait is associated with the $\mathrm{X}: 21$ gene arrangement and there is a large 
variation in its expression, caused by genetic and environmental factors. The sons of SR fathers are almost always fertile, whether they come from progenies containing 50 per cent or a low percentage of males (Carvalho et al., 1989; Carvalho \& Klaczko, 1992, unpublished data). We now report the existence and chromosomal localization of autosomal suppressors of sex-ratio in this species.

\section{Materials and methods}

\section{Strains used in the genetic analysis}

ITA-24-P. A laboratory reference strain with good productivity.

S-50. Homokaryotypic for the $\mathrm{X}: 21$ gene arrangement and routinely produces 50 per cent male progeny. It was obtained by five generations of directional selection for ' 50 per cent males in progeny' applied through brother-sister matings and has been maintained in mass culture since 1988. The suppression of sex-ratio expression in S-50 males appears to be total and their progenies are indistinguishable from those of ST/Y males both in the mean sexual proportion and in the absence of extra binomial variance. The X:21 chromosome of S-50 is functional: its introgression in the ITA24-P autosomal background (by two backcrosses to this strain) restores sex-ratio expression (Carvalho, 1989; Carvalho \& Klaczko, 1992, unpublished data).

$N A$. Homokaryotypic for the $\mathrm{X}: \mathrm{ST}$ gene arrangement and carrying visible markers on all autosomes of $D$. mediopunctata except the dot (chromosome VI): Delta ( $D l$, dominant, chromosome II), Impar (Im, dominant, III), coral ( $\mathrm{cr}$, recessive, IV) and alfinete ( $\mathrm{al}$, recessive, V); (Marques et al., 1991, unpublished data). The strain is heterozygous for $D l$ and $I m$ owing to their recessive lethality.

$N B$. Homokaryotypic for $\mathrm{X}: 21$ and homozygous for coral and alfinete. Preliminary crosses on NB and NA showed that these strains are free from autosomal suppressors of sex-ratio. A cross between NB and S-50 showed that their polytene SR chromosomes are homosequential (these chromosomes were derived from unrelated strains).

\section{Localization of the suppressors with crosses in mass (experiment I)}

As usual in the genus Drosophila, there is no crossing over in D. mediopunctata males (H. Marques, personal communication). This permits the localization of the suppressors of S-50 by crossing it with a strain with one visible marker on each chromosome. We controlled male age because this variable affects sex-ratio expression in D. mediopunctata (Carvalho \& Klaczko, 1992).

The general plan of the experiment is depicted in Fig. 1. From the cross between S-50 males and NA females we collected $F_{1}$ males with the Delta Impar phenotype and crossed them with NB females. These two crosses were made en massae, with at least 30 pairs. Every 4 days we collected and sorted the 16 different $\mathrm{F}_{2}$ males (ranging from wild-type to tetramutants) produced by the later cross. All these $F_{2}$ males were $21 / \mathrm{Y}$ and carried different combinations of autosomes derived from S-50 and from marker strains (see also Table 1, first column). To measure their level of sex-ratio expression, 13-day-old $\mathrm{F}_{2}$ males were crossed for 6 days with females (ITA-24-P strain) using 10 pairs per bottle. After this time flies were transferred to new bottles to oviposit for 6 days before finally being discarded.

To reduce competition we added liquid ferment to the cultures regularly and the progenies produced were sexed and counted until bottle exhaustion. We made several replicates for each of the 16 genotypes and considered only those cultures producing at least 100 flies.

As a control, we tested $21 / \mathrm{Y}$ males from the $\mathrm{S}-50$ strain with the same experimental procedures.

\section{Localization of the suppressors with individual crosses (experiment II)}

To characterize the between male-within genotype variation we essentially repeated the experiment described above, making the last cross with one $21 / \mathrm{Y}$ male and three or four ITA-24-P females. Oviposition time was extended to 15 days and only 10 of the 16 genotypes were tested, the six double mutants being discarded. We made between 24 and 30 replicas for each of the 10 genotypes. Only cultures producing 20 or more flies were considered.

Flies were reared in half-pint bottles with trimeveledon medium (Carvalho et al., 1989) at $16.5^{\circ} \mathrm{C}$.

\section{Results}

\section{Experiment I}

Table 1 (experiment I) presents the averages of the percentage of males produced by each of the 16 genotypes. These data were analysed with a four-way ANOVA, using chromosomes II, III, IV and V as factors and the angular transformation of proportion of 
Fig. 1 Chromosomal localization of suppressor genes. Note that all 16 genotypes of $F_{2}$ males are phenotypically recognizable; these males were tested for sex-ratio expression by crossing them with ITA-24-P females.

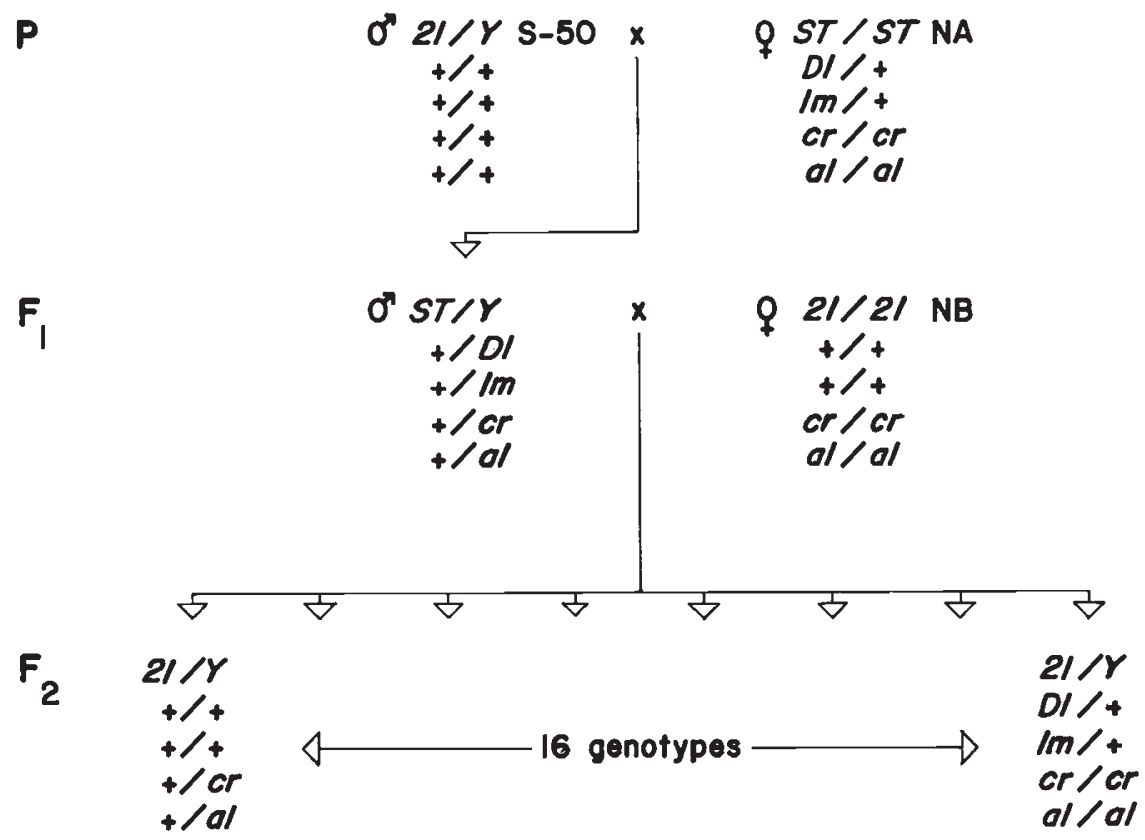

Table 1 Mean sex-ratio expression of males carrying chromosomes derived from S-50 strain (wild-type ones) and from marker strains (mutant ones)

\begin{tabular}{|c|c|c|c|c|c|c|c|}
\hline \multicolumn{4}{|c|}{ Genotype of $F_{2}$ male $\dagger$} & \multicolumn{2}{|c|}{ Experiment I } & \multicolumn{2}{|c|}{ Experiment II } \\
\hline II & III & IV & V & Mean & $N$ & Mean & $N$ \\
\hline+ & + & + & + & 27.7 & 7 & 20.5 & 24 \\
\hline$D l$ & + & + & + & 20.2 & 7 & 13.5 & 22 \\
\hline+ & Im & + & + & 24.8 & 7 & 25.1 & 24 \\
\hline+ & + & $\mathrm{cr}$ & + & 6.1 & 7 & 1.9 & 22 \\
\hline+ & + & + & al & 22.8 & 7 & 13.0 & 23 \\
\hline$D l$ & Im & + & + & 19.2 & 7 & - & - \\
\hline$D l$ & + & $\mathrm{cr}$ & + & 3.6 & 7 & - & - \\
\hline$D l$ & + & + & al & 12.9 & 7 & - & - \\
\hline+ & Im & $\mathrm{cr}$ & + & 4.0 & 7 & - & - \\
\hline+ & $\operatorname{Im}$ & + & al & 25.1 & 7 & - & - \\
\hline+ & + & $c r$ & al & 3.5 & 7 & - & - \\
\hline$D l$ & $I m$ & $\mathrm{cr}$ & + & 6.4 & 7 & 7.8 & 25 \\
\hline$D l$ & Im & + & al & 12.3 & 6 & 13.0 & 25 \\
\hline$D l$ & + & $\mathrm{cr}$ & al & 3.1 & 7 & 2.4 & 26 \\
\hline+ & Im & $c r$ & al & 7.5 & 7 & 5.9 & 28 \\
\hline$D l$ & $I m$ & $\mathrm{cr}$ & al & 4.9 & 6 & 5.6 & 25 \\
\hline \multicolumn{4}{|c|}{$\mathrm{S}-50($ control $)$} & 48.3 & 5 & - & - \\
\hline
\end{tabular}

† Showed only the chromosomes received from the $F_{1}$ males, where segregation occurred.

males $(\operatorname{arc} \sin \downarrow p)$ as the dependent variable. The results of the test are in Table 2: the main effect of chromosomes II, IV and $\mathrm{V}$ was very significant whereas chromosome III had a non-significant effect.
Most of the two-factor interactions were also significant at the 0.05 level; interaction between chromosomes II and IV was very significant. We estimated the mean effect of chromosome II by averaging all genotypes containing Delta and subtracting this from the mean of those that received the S-50 s second chromosome (wild-type). The effect of the other chromosomes was estimated similarly and, as shown in Table 3 (experiment I), chromosome IV had by far the strongest effect: two times greater than the remaining genome.

\section{Experiment //}

The distribution of progenies of individual 21/Y males belonging to the 10 tested genotypes is shown in Fig. 2 and the corresponding means are in Table 1 (experiment II). As in experiment I we applied a four-way ANOVA but, given the lack of data from double mutants, interactions between chromosomes cannot be tested. The effect of all four chromosomes was significant $(P<0.05$ for II; all others below 0.002$)$; their estimates are in Table 3 (experiment II) and were obtained as described above. Chromosome IV again had the greatest effect and this time the 'reverse' effect of III was more evident. This means that chromosome III of the S-50 strain is a weaker suppressor than its homologue from the marker strain.

In all genotypes there was extra binomial variance, as the heterogeneity $G$-test always indicated very significant departures from homogeneity (data not shown). 
Table 2 ANOVA table

\begin{tabular}{lrrrr}
\hline Source & d.f. & \multicolumn{1}{l}{ MS } & \multicolumn{1}{l}{$F$} \\
\hline II & 1 & 0.128 & 31.320 & $* * *$ \\
III & 1 & 0.009 & 2.314 & \\
IV & 1 & 1.738 & 426.618 & $* * *$ \\
V & 1 & 0.036 & 8.926 & $* *$ \\
II $\times$ III & 1 & 0.002 & 0.441 & \\
II $\times$ IV & 1 & 0.060 & 14.664 & $* * *$ \\
II $\times$ V & 1 & 0.018 & 4.526 & $*$ \\
III $\times$ IV & 1 & 0.015 & 3.740 & $*$ \\
III $\times$ V & 1 & 0.017 & 4.055 & $*$ \\
IV $\times$ V & 1 & 0.020 & 4.938 & \\
II $\times$ III $\times$ IV & 1 & 0.003 & 0.784 & $*$ \\
II $\times$ III $\times$ V & 1 & 0.019 & 4.768 & $*$ \\
II $\times$ IV $\times$ V & 1 & 0.002 & 0.601 & \\
III $\times$ IV $\times$ V & 1 & 0.001 & 0.325 & \\
II $\times$ III $\times$ IV $\times$ V & 1 & 0.004 & 0.935 & \\
Error & 94 & 0.004 & & \\
\hline & & & & \\
\hline
\end{tabular}

${ }^{*} P<0.05,{ }^{* *} P<0.01,{ }^{* * *} P<0.001$

\section{Discussion}

Our results demonstrate for the first time the existence of autosomal suppressors compatible with the operation of 'Fisher's Principle' in a species with a SR chromosome. The genetic system we describe is composed of at least four genes (one or more on each major autosome), with expression in the heterozygous state (our experimental design cannot detect recessive suppressors) and strong interactions between them. Chromosome IV had by far the strongest effect.

The interaction between chromosomes deserves some comments. As it is possible that they represent mere scale effects (Falconer, 1989), we tried several transformations, including logarithmic, untransformed proportion of males and probit, the most biologically sound scale for meiotic drive (Miklos \& Smith-White, 1971). In all cases many interactions remained significant (data not shown). Even if the interactions are scale artefacts in the sense that in the physiologically appropriate scale they will disappear, they are real for the operation of natural selection via Fisher's Principle. This occurs because in a population with unequal sex proportions the fitness of an individual is a linear function of the (untransformed) proportion of males produced in the progeny (Bodmer \& Edwards, 1960; Eshel, 1975). So, in the evolution of sexual proportion in $D$. mediopunctata there will exist at least some genetic interaction variance, which does not respond to selection.
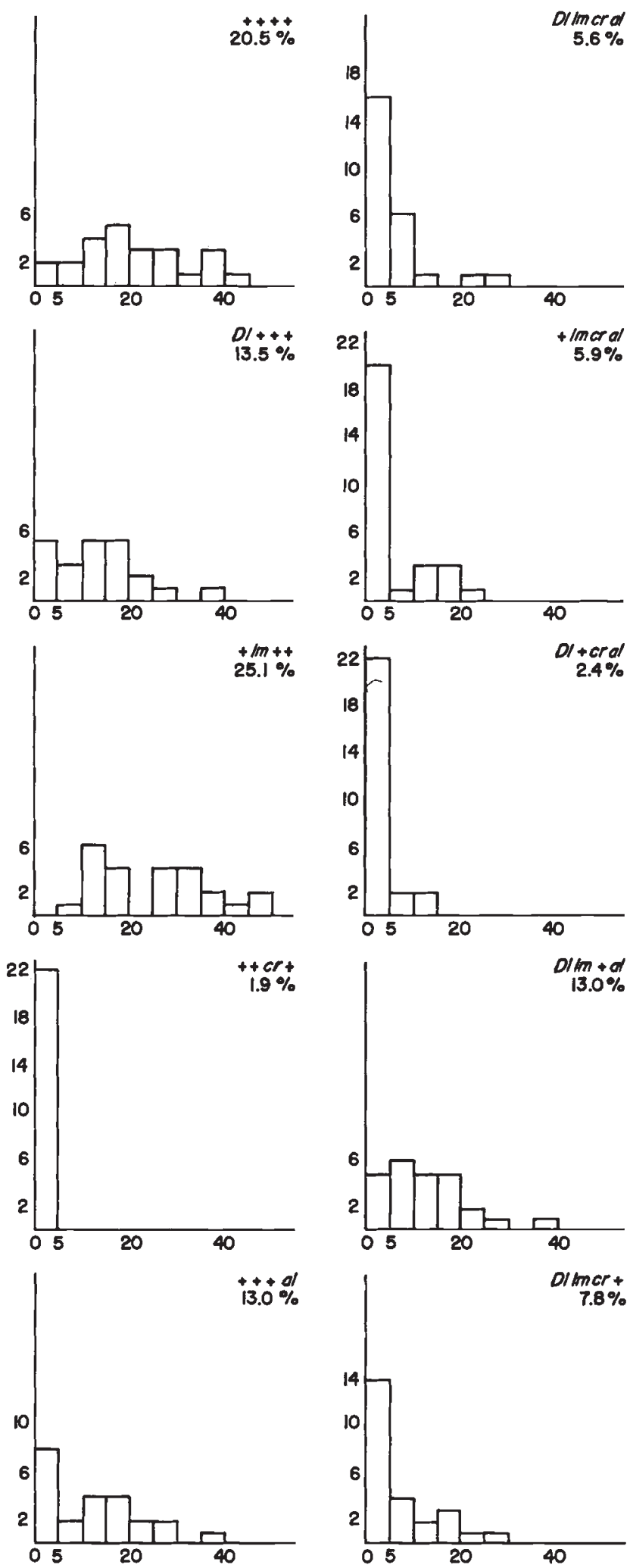

Fig. 2 Distribution of the progenies of $F_{2} 21 / Y$ males in individual crosses. Male genotype and mean sexual proportion are indicated in the corresponding histogram. Abscissae, per cent males in progenies; ordinates, absolute frequency of progenies. 
Table 3 Mean chromosome effect

\begin{tabular}{llllllrl}
\hline & \multicolumn{2}{l}{ Experiment I } & & & \multicolumn{2}{l}{ Experiment II } & \\
\cline { 2 - 3 } \cline { 7 - 8 } Chromosome & S-50 & Marker & Mean effect & & S-50 & Marker & Mean effect \\
\hline II & 15.2 & 10.3 & +4.9 & & 13.3 & 8.5 & +4.8 \\
III & 12.5 & 13.0 & -0.5 & & 10.3 & 11.5 & -1.2 \\
IV & 20.6 & 4.9 & +15.7 & & 17.0 & 4.7 & +12.3 \\
V & 14.0 & 11.5 & +2.4 & & 13.8 & 8.0 & +5.8 \\
\hline
\end{tabular}

Columns 2 and 5, means of genotypes carrying S-50 chromosomes; columns 3 and 6 , means of genotypes with chromosomes from the NA marker strain.

Experiment II showed that there was variation in sex-ratio expression between males sharing the same chromosomal genotype. This extra binomial variance (which was almost never observed in ST/Y males; Carvalho et al., 1989) may be caused by environmental factors such as density, minor temperature or age variation or by residual genetic variation, as the segregation of the dot chromosome (VI) was not followed and neither S-50 nor the marker strains are isogenic. These same factors (plus the differences in experimental design) may be responsible for the small discrepancies between experiments I and II (see Tables $1,3)$.

As usual in genetic analysis, some caution is necessary concerning the generality of our results. The major effect of chromosome IV and the interactions and dominance relations we found are strictly valid only for the strains and experimental conditions we used. In fact S-50 is totally dominant in crosses with a strain selected for 20 per cent males (data not shown).

The occurrence of autosomal suppressors has implications for the evolution of the sex-ratio system in D. mediopunctata. Assuming that the X:21 polymorphism in this species results from an equilibrium between meiotic drive and natural selection (as occurs in D. pseudoobscura: Wallace, 1948; Curtsinger, 1991), these suppressors probably contribute to stabilize the frequency of $\mathrm{X}: 21$ at its present level ( 10 per cent; see Carvalho et al., 1989) because they reduce the meiotic drive advantage of this chromosome.

Our results demonstrate the existence of autosomal suppressors compatible with the operation of Fisher's Principle in $D$. mediopunctata. The study of populations in the laboratory may show if they can indeed respond (as expected) to this kind of selection whereas field studies (for example, the geographical distribution of suppressors and X:21 chromosomes) may, perhaps, indicate if this process has been operating in natural populations of D. mediopunctata. We are now trying to answer both questions.

\section{Acknowledgements}

This work was partially supported by Conselho Nacional de Desenvolvimento Científico e Tecnólogico-CNPq and Coordenação de Aperfeiçoamento do Pessoal de Ensino Superior-CAPES. We thank Dr A. M. Solé-Cava, S. F. Reis, A. N. Hagler, J. C. R. Baptista, B. C. Bitner-Mathé and an anonymous referee for valuable suggestions in the manuscript; Ms Mônica Bahia Schlee and Mr Octavio de Pontes for graphical assistance; Ms Cléa Knauer da Silva and Natalícia dos Santos for technical assistance; Mr H. V. S. Marques for some mutant strains and Dr L. De Meis for his kind help. We also thank Tintas Ypiranga S.A. for a donation to our laboratory.

\section{References}

BeCKenbaCH, A. T. 1991. Sex-ratio polymorphism in Drosophila pseudoobscura. Am. Nat., 137, 340-343.

BECKENBACH, A.T., CURTSINGER, J. W. AND POLICANSKY, D. 1982. Fruitless experiments with fruit flies: the 'sex-ratio' chromosomes of D. pseudoobscura. Drosophila Inform. Serv., 58, 22.

BODMER, W. F. AND EDWARDS, A. W. F. 1960. Natural selection and the sex ratio. Ann. Hum. Genet., 24, 239-244.

CARvalho, A. B. 1989. Sex-ratio em Drosophila mediopunctata. M.Sc. Thesis, Universidade Federal do Rio de Janeiro.

CARVAlHo, A. B. AND KLACZKo, L. B. 1992. Age and sex-ratio expression in Drosophila mediopunctata. Genetica, 87, $107-111$.

CARvalho, A. B., PEIXoto, A. A. AND klaczko, L. B. 1989. Sexratio in Drosophila mediopunctata. Heredity, 62 , 425-428.

COBBS, G. 1986. An investigation of the genetics of the 'male sex-ratio' in Drosophila pseudoobscura. Genetics, 113, 355-365.

CoBBs, G. 1987. Modifier genes of the sex-ratio trait in Drosophila pseudoobscura. Genetics, 116, 275-283. 
COBBS, G., JEWELL, L. AND GORDON, L. 1991. Male sex-ratio trait in Drosophila pseudoobscura: frequency of autosomal aneuploid sperm. Genetics, 127, 381-390.

CONOVER, D. O. AND VOORHEES, D. A. 1990. Evolution of a balanced sex ratio by frequency-dependent selection in a fish. Science, 250, 1556-1558.

CURTSINGER, J. w. 1991. X-chromosome segregation distortion in Drosophila. Am. Nat., 137, 344-348.

ESHEL, I. 1975. Selection on sex-ratio and the evolution of sex-determination. Heredity, 34, 351-361.

FALCONER, D. S. 1989. Introduction to Quantitative Genetics, 3rd edn. Longman, London, Chap. 17.

FISHER, R. A. 1930. The Genetical Theory of Natural Selection. Clarendon Press, Oxford.

GERSHENSON, s. 1928. A new sex-ratio abnormality in $D$. obscura. Genetics, 13, 488-507.

hamilton, w. D. 1967. Extraordinary sex ratios. Science, 156, $477-488$.

HAUSCHTECK-IUNGEN, E. AND MAURER, B. 1976. Sperm dysfunction in sex-ratio males of Drosophila subobscura. Genetica, 46, 459-477.

JAMES, A. C. AND JAENIKE, J. 1990. 'Sex-ratio' meiotic drive in Drosophila testacea. Genetics, 126, 651-656.
MARQUES, H. V. S., CARVALHO, A. B., ELIAS, C. A. AND KLACZKo, L. B. 1991. Mutants of D. mediopunctata. Drosophila Inform. Serv., 70, 280.

MIKLOS, G. L. G. AND SMITH-WhITE, S. 1971. An analysis of the instability of Segregation-Distorter in Drosophila melano gaster. Genetics, 67, 305-317.

POLICANSKY, D. AND DEMPSEY, B. 1978. Modifiers and 'sex-ratio' in Drosophila pseudoobscura. Evolution, 32, 922-924.

POLICANSKY, D. AND ELLISON, J. 1970. 'Sex-ratio' in Drosophila pseudoobscura: spermiogenic failure. Science, 169, 888-889.

STALKER, H. D. 1961. The genetic systems modifying meiotic drive in Drosophila paramelanica. Genetics, 46, 177-202.

THOMSOM, G. J. AND FELDMAN, M. W. 1975. Population genetics of modifiers of meiotic drive: IV. On the evolution of sexratio distortion. Theor. Pop. Biol., 8, 202-211.

VOELKER, R. A. 1972. Preliminary chracterization of 'sex-ratio' and reinterpretation of 'male sex-ratio' in Drosophila affinis. Genetics, 71, 597-606.

WALlace, B. 1948. Studies on 'sex-ratio' in Drosophila pseudoobscura. I. Selection and 'sex-ratio'. Evolution, 2, 189-217. 